\title{
Implementação de sistemas de gestão de acordo com o ciclo de vida organizacional: uma revisão sistemática da literatura
}

\author{
RESUMO
}

\section{Rodrigo Salgado Sátiro} rssatiro@gmail.com

Faculdade de Tecnologia Ciências e Educação (FATECE), Pirassununga, SP Brasi

Sheyla Mara Baptista Serra sheylabs@ufscar.br

Universidade Federal de São Carlos (UFSCar), São Carlos, SP, Brasil
Uma das formas de analisar determinado assunto na academia é por meio de uma Revisão Sistemática da Literatura (RSL). Existem vários métodos que apresentam um processo estruturado que permite identificar lacunas de conhecimento de acordo com as definições que foram feitas para esse tipo de pesquisa sobre o tema em questão. 0 objetivo deste trabalho é analisar referências científicas sobre a implantação de sistemas de gestão empresarial de acordo com o ciclo de vida organizacional. Para isso, utilizou o método Proknow-C, para pesquisar e analisar quantitativamente artigos relevantes para esse tema. As definições das bases de dados foram realizadas no mês de dezembro de 2016. Foram identificados 6.311 artigos, sendo selecionados 30 artigos mais relevantes, publicados entre os anos de 1979 a 2012. A análise das publicações evidenciou que existe potencial para continuidade das pesquisas em assuntos discutidos há anos, como a Teoria do Ciclo de Vida Organizacional, e novos temas correlacionados, como a Mensuração do Desempenho Organizacional e a Sistematização das Antecipações Gerenciais.

PALAVRAS-CHAVE: Implantação de Sistemas de Gestão. Ciclo de Vida Organizacional. Revisão Sistemática da Literatura. RSL. ProKnow-C. 


\section{INTRODUÇÃO}

A Revisão Sistemática da Literatura (RSL) é um método utilizado para analisar o que já foi publicado sobre determinado assunto e que permite identificar lacunas de conhecimento. Esse tipo de pesquisa pode ser definido como uma síntese de estudos primários que contém objetivos, materiais e métodos claramente explicitados e que foi conduzida de acordo com uma metodologia clara e reprodutível (Greenhalgh, 1997).

Diversos trabalhos foram publicados para identificação, constatação e direcionamento de pesquisas para o avanço do conhecimento na área de administração. Por exemplo, Tomomitsu et al. (2018) pesquisaram a relação entre gestão de projetos e gestão do conhecimento; Silveira e Petrini (2018) apresentaram estudos bibliométricos sobre temáticas relacionadas com desenvolvimento sustentável e responsabilidade social corporativa; e Morioka, et. al. (2018) fizeram uma revisão sistemática da literatura sobre sistemas de mensuração de desempenho (SMD), com foco na sustentabilidade.

Assim, verifica-se como oportunidade de realizar uma RSL para entender o contexto do ciclo de vida organizacional em relação à implantação de sistemas de gestão empresarial. Observou-se que dentro do próprio tema não há um consenso sobre a estrutura dos ciclos de vida organizacional.

Os estudos do ciclo de vida organizacional remontam a década de sessenta com a publicação de Lippitt e Schimidt (1967) e até o presente momento existem autores pesquisando este assunto, como pode-se identificar em Cantner e Wolf (2018), Li e Zhang (2018) e Rocco e Manna (2018).

Adizes (1979) propôs um modelo de ciclo de vida organizacional onde menciona que as empresas passam por estágios recorrentes, que seguem os dez estágios da vida humana: namoro, infância, pré-adolescência, adolescência, plenitude, estabilidade, aristocracia, burocracia insipiente, burocracia e morte.

Para Phelps et al. (2007) não há uma sequência lógica nem tampouco linear para que as empresas avancem ou sigam nos estágios do ciclo de vida. Há na verdade um conjunto de problemas e questões chaves em cada momento do seu desenvolvimento que as empresas terão que resolver para avançar ou para se manterem vivas. Esses momentos são definidos pelos autores como pontos de inflexão, em que o crescimento ocorre pela assimilação do conhecimento e soluções para avançar por esses pontos de inflexão.

Corroborando Whetten (1987) menciona que o conceito de estágios não deve estar atrelado ao conceito de desenvolvimento dos seres vivos e sim deve ser interpretado livremente como um conjunto de problemas que uma organização irá enfrentar para seguir para outros estágios. $O$ autor complementa que esse tipo de abordagem de rastreamento, enfrentamento e solução de problemas parece ser mais adequado uma vez que trata no nível de subsistemas das organizações.

Para Achtenhagen et al. (2010) há grande divergência sobre a percepção de crescimento da empresa entre os pesquisadores e os empreendedores. Seu estudo comprovou que as variáveis utilizadas pelos pesquisadores e como são mensuradas não refletem o que os empreendedores analisam para afirmar se uma empresa está crescendo ou não. 
Levie e Lichtenstein (2010) propõem uma alternativa à teoria do ciclo de vida organizacional, sendo esta, o estado dinâmico, que deve analisar o empreendedor, sua forma de organização e o nicho de mercado. A eficácia e a eficiência de um modelo podem ser medidas pelo valor criado por uma organização. Observa-se, assim, que existem estudos sobre as fases iniciais do ciclo de vida organizacional e as composições de elementos de cada fase, porém existem lacunas em relação ao gerenciamento do crescimento e ao declínio das empresas (Whetten, 1987), bem como a relação com novos enfoques administrativos. Dada a relevância do assunto e a existência de diferentes abordagens teóricas, o objetivo deste artigo é realizar uma RSL sobre a implementação de sistemas de gestão empresarial de acordo com o ciclo de vida organizacional e identificar as oportunidades de pesquisa conforme os critérios de análise definidos.

\section{MÉTODO DE PESQUISA}

Para Vilela (2012), com o advento das bases de dados compartilhadas pela internet e consequente disponibilidade de grande quantidade de artigos científicos, uma das dificuldades do pesquisador é encontrar aqueles que são de maior relevância para o tema que se deseja pesquisar.

O método selecionado para a RSL foi o ProKnow - Knowledge Development Process-Constructivist, proposto por Ensslin et al. (2010), devido apresentar um roteiro para realização da referida pesquisa com as etapas muito bem definidas, estruturadas. De acordo com os autores do método, este possui as seguintes etapas: Definições da pesquisa; Seleção dos artigos; e Análise bibliométrica. Cada etapa se subdivide em subetapas, como pode ser verificado no Quadro 1, que apresenta as etapas do método ProKnow-C e suas ramificações.

Quadro 1 - Método ProKnow-C para revisão sistemática da literatura

\begin{tabular}{|c|c|}
\hline Etapas & Subetapas \\
\hline \multirow{3}{*}{ 1. Definições da Pesquisa } & 1.1. Cronologia da Pesquisa \\
\hline & 1.2 Bases de dados \\
\hline & 1.3. Filtros de pesquisa \\
\hline \multirow{9}{*}{ 2. Seleção dos Artigos } & 2.1. Validação das Bases de Artigos \\
\hline & 2.1. Seleção dos Artigos \\
\hline & 2.2. Teste de aderência das palavras-chave \\
\hline & 2.3. Eliminação de redundâncias \\
\hline & 2.4. Alinhamento do título do artigo \\
\hline & 2.5. Reconhecimento científico \\
\hline & 2.6. Alinhamento do resumo do artigo \\
\hline & 2.7. Reavaliação dos artigos menos citados \\
\hline & 2.8. Análise final \\
\hline \multirow{4}{*}{$\begin{array}{l}\text { 3. Análise Bibliométrica do } \\
\text { Portfólio Final dos Artigos }\end{array}$} & 3.1. Estimar o grau de relevância dos periódicos \\
\hline & 3.2. Estimar o reconhecimento científico dos artigos \\
\hline & 3.3. Estimar o grau de relevância dos autores \\
\hline & 3.4. Determinar as palavras-chaves mais usadas \\
\hline
\end{tabular}

Fonte: Autoria Própria (2018), baseado em Ensslin et al. (2010) 
Vilela (2012) menciona que a escolha do ProKnow-C se justifica na simplicidade e sistematização da atividade para a construção do conhecimento necessário para iniciar uma pesquisa acadêmica. A seguir são apresentadas as etapas seguidas para o desenvolvimento desta pesquisa.

\section{DEFINIÇÕES DA PESQUISA}

Na etapa "Definições da Pesquisa" é definido o que será pesquisado, delimita-se o período em que será realizada a pesquisa e quais os filtros a serem utilizados na mesma. No Quadro 2 é apresentado um resumo dessa etapa.

Quadro 2 - Definições da pesquisa

\begin{tabular}{|c|c|}
\hline Definições & Detalhamento \\
\hline Cronologia da Pesquisa & Janeiro à Abril de 2017 \\
\hline Bases de Dados & Portal de Periódicos Capes \\
& Scielo \\
& Science Direct \\
& Willey Online Library \\
& Web of Science \\
\hline Filtros de Pesquisa & Organizational Longevity \\
(Title, Abstract and Key-words) & Organizational Maturity \\
& Organizational Lifecycle \\
& Organizational Life Cycle \\
& Implementation of Management System \\
& Stages of Growth .and. Business \\
& Stages of Growth .and. Organizational \\
\hline Tipo de Documento & Artigos \\
\hline
\end{tabular}

Fonte: Autoria própria (2018)

\section{Cronologia da Pesquisa}

As definições das bases de dados foram realizadas no mês de dezembro de 2016; a seleção dos artigos nas Bases de Dados foi realizada entre os meses de janeiro e março de 2017.

\section{Bases de Dados}

Para este trabalho optou-se por considerar todas as bases de dados disponíveis para consulta na Biblioteca Comunitária da Universidade Federal de São Carlos (UFSCar), com pesquisas nas áreas de engenharias e ciências sociais aplicadas, ciências contábeis, economia, administração e ciências sociais), sendo elas: Portal de Periódicos CAPES, Science Direct, Willey Online Library, Web of Science e Scielo.

\section{Filtros de Pesquisa}

Nos filtros de pesquisa são definidos o período da pesquisa, as palavraschave, tipos de documentos e itens dos documentos que serão pesquisados. 
Não foi realizado um recorte do período de pesquisa, uma vez que a proposta foi analisar toda a produção realizada até a presente data sobre o assunto da pesquisa. As palavras-chave de pesquisa foram definidas em torno de dois eixos de pesquisa: ciclo de vida organizacional e implementação de sistemas de gestão.

No eixo ciclo de vida organizacional, foram definidos inicialmente as palavras-chave: "Organizational Lifecycle" e sua variação "Organizational Life Cycle" e "Organizational Maturity". Para garantir que os principais termos fossem utilizados no momento das buscas foi realizada uma consulta ao Tesauro que além das palavras chaves já definidas apresentou também o termo "Organizational Longevity".

$\mathrm{Na}$ fase do teste de aderência das palavras-chave, item - Seleção dos artigos, foram identificadas duas novas-palavras chave que foram incorporadas à busca "Stages of Growth .and. Business" e "Stages of Growth .and. Organizational", inclusão esta que será apresentada no referido item.

No eixo implementação de sistemas de gestão foi utilizada como palavrachave "Implementation of Management System".

Para os tipos de documentos a serem pesquisados optou-se por utilizar somente artigos científicos revisados por pares quando o portal proporcionava esse tipo de seleção.

\section{SELEÇÃO DOS ARTIGOS}

As atividades referentes à etapa de seleção dos artigos foram realizadas no período de 06 de janeiro de 2017 à 03 de março de 2017.

\section{Validação das bases de artigos}

Utilizando as palavras-chave definidas na fase de definição da pesquisa, o processo de busca nas bases de dados retornou um total de 3201 artigos.

\section{Seleção dos Artigos}

Os artigos foram extraídos para o gerenciador de referências Mendeley Desktop versão 1.17.9.

Quando o mecanismo de busca da base de dados permitiu foi realizada a primeira seleção excluindo áreas não relacionadas com Administração ou Engenharias.

\section{Teste de aderência das Palavras-Chave}

Após a fase da carga no gerenciador de referências Mendeley Desktop, realizou-se o teste de aderência das palavras-chave utilizadas, selecionou-se dois artigos com grande quantidade de citações e que estavam alinhados no título e resumo com o tema da pesquisa e analisou-se as palavras-chave desses artigos 
identificando-se mais uma palavra-chave não utilizada na busca inicial: "Stage of Growth".

Neste momento, realizou-se nova busca utilizando o termo "Stage of Growth" que retornou uma quantidade muito grande de artigos, principalmente relacionados à Botânica e à Pediatria. Dessa forma, utilizou-se combinado com a palavra-chave os termos "Bussiness" e "Organizational", o que resultou em mais 3760 artigos, totalizando 6961 artigos.

Novamente selecionou-se dois novos artigos com grande quantidade de citações, analisou-se o título e o resumo que se apresentaram alinhados com o tema da pesquisa e verificou-se que todas as palavras-chave estavam incorporadas, considerando-se finalizado o processo de busca de artigos.

\section{Eliminação de Redundâncias}

Com o auxílio do gerenciador de referências Mendeley Desktop, realizou-se o processo de identificação de duplicatas de artigos, duplicatas essas que ocorrem normalmente devido a indexação de periódicos por mais de uma base de dados ou devido a um artigo atender a mais de uma palavra-chave, conforme recomendado pelo método Proknow-C (Ensslin et al., 2010).

Também foram excluídos documentos que não fossem artigos que o mecanismo de busca da base de dados não permitiu selecionar no processo de extração dos documentos. Após a eliminação das duplicatas restaram 6311 artigos, conforme Quadro 3.

Quadro 3-Quantidade de artigos por Bases de Dados

\begin{tabular}{|c|c|c|c|c|c|c|c|c|}
\hline String & $\begin{array}{c}\text { Web of } \\
\text { Science }\end{array}$ & $\begin{array}{c}\text { Portal } \\
\text { CAPES }\end{array}$ & Willey & $\begin{array}{c}\text { Science } \\
\text { Direct }\end{array}$ & Scielo & Total & $\begin{array}{c}\text { Dupli- } \\
\text { catas }\end{array}$ & Final \\
\hline $\begin{array}{c}\text { Implementation } \\
\text { of Management } \\
\text { Systems }\end{array}$ & 9 & 18 & 48 & 68 & 2 & 145 & 11 & 134 \\
\hline $\begin{array}{c}\text { Organizational } \\
\text { Life Cycle }\end{array}$ & 55 & 775 & 584 & 385 & 10 & 1809 & 307 & 1502 \\
\hline $\begin{array}{c}\text { Organizational } \\
\text { Lifecycle }\end{array}$ & 3 & 45 & 31 & 82 & 2 & 163 & 21 & 142 \\
\hline $\begin{array}{c}\text { Organizational } \\
\text { Longevity }\end{array}$ & 11 & 98 & 41 & 20 & 1 & 171 & 26 & 145 \\
\hline $\begin{array}{c}\text { Organizational } \\
\text { Maturity }\end{array}$ & 40 & 468 & 165 & 234 & 6 & 913 & 156 & 757 \\
\hline $\begin{array}{c}\text { Stages of Growth } \\
\text {.and. Business }\end{array}$ & 42 & 329 & 1155 & 944 & 0 & 2470 & 77 & 2393 \\
\hline $\begin{array}{c}\text { Stages of Growth } \\
\text { and. } \\
\text { Organizational }\end{array}$ & 31 & 50 & 676 & 531 & 2 & 1290 & 52 & 1238 \\
\hline \begin{tabular}{c} 
Total \\
\hline
\end{tabular}
\end{tabular}

Fonte: Autoria própria (2018) 
Na fase de alinhamento do título é realizada a leitura dos títulos de cada artigo para validação do alinhamento deste com a pesquisa. Esse processo resultou num total de 394 artigos.

\section{Reconhecimento Científico}

A análise do reconhecimento científico é realizada com base no número de citações dos artigos. Estabeleceu-se um valor para corte de $85 \%$ do número de citações dos artigos, uma vez que o método utilizado para este trabalho, Ensslin et al. (2010) não define um valor de corte. Entretanto, procurou-se analisar uma amostra significativa das publicações selecionadas.

A soma total das citações dos 394 artigos foi de 26.024 citações. Utilizando o critério de corte de $85 \%$, selecionou-se artigos com 67 ou mais citações, o que correspondeu a um total de 84 artigos.

Os demais 310 artigos foram reavaliados na etapa "Reavaliação dos artigos menos citados", de acordo com os critérios desta etapa e puderam ser selecionados para o portfólio final.

\section{Alinhamento do Resumo do Artigo}

Os 84 artigos selecionados na etapa anterior foram analisados em função do alinhamento do seu resumo com o tema da pesquisa, sendo que destes, 46 foram excluídos devido ao não alinhamento com o tema da pesquisa, restando 38 artigos com o título e resumo alinhados com o tema da pesquisa, conforme Quadro 4.

Quadro 4 - Análise dos temas dos artigos do Portfólio

\begin{tabular}{|c|c|}
\hline Tipo de Abordagem sobre o Ciclo de Vida Organizacional & Quantidade \\
\hline Teoria do Ciclo de Vida Organizacional & 08 \\
\hline $\begin{array}{c}\text { Estudo de aspecto organizacional utilizando a Teoria do } \\
\text { Ciclo de Vida Organizacional }\end{array}$ & 13 \\
\hline Implantação de Sistemas de Gestão Certificado & 03 \\
\hline Fora de Escopo & 06 \\
\hline Não disponível & 04 \\
\hline Empreendedorismo & 01 \\
\hline Alianças Estratégicas & 01 \\
\hline Modelo de crescimento de empresas de capital de risco & 02 \\
\hline TOTAL & $\mathbf{3 8}$ \\
\hline
\end{tabular}

Fonte: Autoria própria (2018)

\section{Reavaliação dos Artigos menos citados}

Nesta etapa do processo da pesquisa foram avaliados os artigos menos citados que atendiam aos dois critérios simultaneamente, conforme recomendado pelo método Proknow-C (Ensslin et al., 2010): 
a) artigos publicados há menos de dois anos,

b) artigos com autoria de um dos pesquisadores presentes no grupo dos 38 artigos selecionados na etapa anterior.

Nesta etapa não foi encontrado nenhum artigo com menos de dois anos que fosse de autoria de, pelo menos, de um dos pesquisadores.

\section{Análise Final}

$\mathrm{Na}$ análise final, é realizada a leitura integral dos artigos selecionados nas etapas anteriores o que ocorreu com os 38 artigos selecionados para identificar os artigos que apresentem modelos de ciclo de vida organizacional ou de implementação de sistemas de gestão.

A leitura dos 38 artigos resultou numa seleção final de 30 artigos para o Portfólio Final de Artigos, conforme apresentado no Quadro 5. Este quadro apresenta os artigos que irão compor o portfólio final de artigos, compreendendo os artigos que compõe a Teoria do Ciclo de Vida Organizacional, Estudo de aspecto organizacional utilizando a Teoria do Ciclo de Vida Organizacional e Implantação de Sistemas de Gestão Certificados.

Quadro 5-Análise Final dos Artigos do Portfólio Bibliográfico

\begin{tabular}{|c|c|c|c|c|c|}
\hline $\mathbf{N}^{\circ}$ & Autor & Título & Periódico & Ano & Citações \\
\hline 1 & $\begin{array}{l}\text { Achtenhagen, L.; } \\
\text { Naldi, L.; Melin, } \\
\text { L.. }\end{array}$ & $\begin{array}{l}\text { "Business Growth": do } \\
\text { practitioners and scholars } \\
\text { really talk about the same } \\
\text { thing? }\end{array}$ & $\begin{array}{c}\text { Entrepreneurship } \\
\text { Theory and } \\
\text { Practice, 34(2): } \\
\text { p.289-316. } \\
\end{array}$ & 2010 & 201 \\
\hline 2 & Adizes, I. & $\begin{array}{c}\text { Organizational passages- } \\
\text { diagnosing and treating } \\
\text { lifecycle problems of } \\
\text { organizations }\end{array}$ & $\begin{array}{c}\text { Organizational } \\
\text { Dynamics, 8(1): } \\
\text { p.3-25 }\end{array}$ & 1979 & 712 \\
\hline 3 & $\begin{array}{l}\text { Anthony e } \\
\text { Ramesh }\end{array}$ & $\begin{array}{c}\text { Association between } \\
\text { accounting performance } \\
\text { measures and stock prices: } \\
\text { a test of the life cycle } \\
\text { hypothesis }\end{array}$ & $\begin{array}{l}\text { Journal of } \\
\text { Accounting and } \\
\text { Economics, } 15(2- \\
\text { 3): } 203-227\end{array}$ & 1992 & 642 \\
\hline 4 & $\begin{array}{c}\text { Auzair, S.M.; } \\
\text { langfield-Smith, } \\
\text { K. }\end{array}$ & $\begin{array}{l}\text { The effect of service } \\
\text { process type, business } \\
\text { strategy and life cycle stage } \\
\text { on bureaucratic MCS in } \\
\text { service organizations }\end{array}$ & $\begin{array}{c}\text { Management } \\
\text { Accounting } \\
\text { Research, 16(4): } \\
\text { p.339-421 }\end{array}$ & 2005 & 201 \\
\hline 5 & $\begin{array}{l}\text { Balkin, D.B.; } \\
\text { Montemayor, } \\
\text { E.F. }\end{array}$ & $\begin{array}{c}\text { Explaining team-based pay: } \\
\text { a contingency perspective } \\
\text { based on the organizational } \\
\text { life cycle, team design, and } \\
\text { organizational learning } \\
\text { literatures }\end{array}$ & $\begin{array}{l}\text { Human Resource } \\
\text { Management } \\
\text { Review, 10(3): } \\
\text { p.249-269 }\end{array}$ & 2000 & 67 \\
\hline 6 & $\begin{array}{l}\text { Beatty, R.W.; } \\
\text { Ulrich, D.O. }\end{array}$ & $\begin{array}{c}\text { Re-energizing the mature } \\
\text { organization }\end{array}$ & $\begin{array}{c}\text { Organizational } \\
\text { Dynamics, } 20(1): \\
\text { p.16-30 }\end{array}$ & 1991 & 102 \\
\hline 7 & Ben-ner, A. & $\begin{array}{l}\text { The life cycle of worker- } \\
\text { owned firms in market } \\
\text { economies: a theoretical }\end{array}$ & $\begin{array}{c}\text { Journal of } \\
\text { Economic Behavior } \\
\text { \& Organization, }\end{array}$ & 1988 & 175 \\
\hline
\end{tabular}




\begin{tabular}{|c|c|c|c|c|c|}
\hline $\mathbf{N}^{\circ}$ & Autor & Título & Periódico & Ano & Citações \\
\hline & & analysis & 10(3): p.283-313 & & \\
\hline 8 & $\begin{array}{l}\text { Beverland, M.; } \\
\text { Lockshin, L.S. }\end{array}$ & $\begin{array}{l}\text { Organizational life cycles in } \\
\text { small New Zealand wineries }\end{array}$ & $\begin{array}{l}\text { Journal of Small } \\
\text { Business } \\
\text { Management, } \\
\text { 39(4): p.354-362 }\end{array}$ & 2001 & 121 \\
\hline 9 & Bredillet, C. & $\begin{array}{c}\text { Genesis and role of } \\
\text { standards: theoretical } \\
\text { foundations and socio- } \\
\text { economical model for the } \\
\text { construction and use of } \\
\text { standards }\end{array}$ & $\begin{array}{l}\text { International } \\
\text { Journal of Project } \\
\text { Management, } \\
\text { 21(6): p.463-470 }\end{array}$ & 2003 & 68 \\
\hline 10 & $\begin{array}{l}\text { Dibrell, C.; Craig, } \\
\text { J.; Hansen, E. }\end{array}$ & $\begin{array}{l}\text { Natural environment, } \\
\text { market orientation, and } \\
\text { firm innovativeness: an } \\
\text { organizational life cycle } \\
\text { perspective }\end{array}$ & $\begin{array}{l}\text { Journal of Small } \\
\text { Business } \\
\text { Management, } \\
\text { 49(3): p.467-489 }\end{array}$ & 2011 & 76 \\
\hline 11 & $\begin{array}{l}\text { Filatotchev, I.; } \\
\text { Toms, S.; } \\
\text { Wright, M. }\end{array}$ & $\begin{array}{l}\text { The firms strategic } \\
\text { dynamics and corporate } \\
\text { governance life- cycle }\end{array}$ & $\begin{array}{l}\text { International } \\
\text { Journal of } \\
\text { Managerial } \\
\text { Finance, 2(4): } \\
\text { p.256-279 }\end{array}$ & 2006 & 139 \\
\hline 12 & $\begin{array}{l}\text { Granlund, M.; } \\
\text { Taipaleenm, J. }\end{array}$ & $\begin{array}{l}\text { Management control and } \\
\text { controllership in new } \\
\text { economy firms: a life cycle } \\
\text { perspective }\end{array}$ & $\begin{array}{c}\text { Management } \\
\text { Accounting } \\
\text { Research, 16(1): } \\
\text { p.21-57 }\end{array}$ & 2005 & 186 \\
\hline 13 & $\begin{array}{c}\text { Hanks, S.; } \\
\text { Watson, C.J.; } \\
\text { Jansen, E.; } \\
\text { Chandler, G. N. }\end{array}$ & $\begin{array}{l}\text { Tightening the life-cycle } \\
\text { construct: a taxonomic } \\
\text { study of growth stage } \\
\text { configurations in high- } \\
\text { technology organizations }\end{array}$ & $\begin{array}{l}\text { Entrepreneurship: } \\
\text { Theory and } \\
\text { Practice } 18(2): \text { p.5- } \\
29\end{array}$ & 1994 & 789 \\
\hline 14 & $\begin{array}{l}\text { Hite, J.M.; } \\
\text { Hesterly, W.S. }\end{array}$ & $\begin{array}{l}\text { The evolution of firm } \\
\text { networks: from emergence } \\
\text { to early growth of the firm }\end{array}$ & $\begin{array}{c}\text { Strategic } \\
\text { Management } \\
\text { Journal, 22(3): } \\
\text { p.275-286 } \\
\end{array}$ & 2001 & 1393 \\
\hline 15 & Holdsworth, R. & $\begin{array}{l}\text { Practical applications } \\
\text { approach to design, } \\
\text { development and } \\
\text { implementation of an } \\
\text { integrated management } \\
\text { system }\end{array}$ & $\begin{array}{l}\text { Journal of } \\
\text { Hazardous } \\
\text { Materials, 104(1- } \\
\text { 3): p.193-205 }\end{array}$ & 2003 & 78 \\
\hline 16 & Hoy, F. & $\begin{array}{c}\text { The complicating factor of } \\
\text { life cycles in corporate } \\
\text { venturing }\end{array}$ & $\begin{array}{l}\text { Entrepreneurship: } \\
\text { Theory and } \\
\text { Practice, 30(6): } \\
\text { p.831-836 } \\
\end{array}$ & 2006 & 90 \\
\hline 17 & $\begin{array}{l}\text { Jawahar, I.M.; } \\
\text { McLaughlin, G.L. }\end{array}$ & $\begin{array}{c}\text { Toward a descriptive } \\
\text { stakeholder theory: an } \\
\text { organizational life cycle } \\
\text { approach }\end{array}$ & $\begin{array}{c}\text { Academic of } \\
\text { Management } \\
\text { Review, 26(3): } \\
\text { p.397-414 }\end{array}$ & 2001 & 1118 \\
\hline 18 & $\begin{array}{l}\text { Karapetrovic, S.; } \\
\text { Casadesús, M. }\end{array}$ & $\begin{array}{c}\text { Implementing } \\
\text { environmental with other } \\
\text { standardized management } \\
\text { systems: scope, sequence, } \\
\text { time and integration } \\
\end{array}$ & $\begin{array}{l}\text { Journal of Cleaner } \\
\text { Production, } 17(5) \text { : } \\
\text { p.533-540 }\end{array}$ & 2009 & 163 \\
\hline 19 & Kallunki, J.P; & The effect of organizational & Management & 2008 & 144 \\
\hline
\end{tabular}




\begin{tabular}{|c|c|c|c|c|c|}
\hline $\mathbf{N}^{\circ}$ & Autor & Título & Periódico & Ano & Citações \\
\hline & Silvola, H. & $\begin{array}{l}\text { life cycle stage on the use of } \\
\text { activity-based costing }\end{array}$ & $\begin{array}{c}\text { Accounting } \\
\text { Research, 19(1): } \\
\text { p.62-79 }\end{array}$ & & \\
\hline 20 & $\begin{array}{l}\text { Leitch, C.; Hill, } \\
\text { F.; Neergaard, H. }\end{array}$ & $\begin{array}{c}\text { Entrepreneurial and } \\
\text { business growth and the } \\
\text { quest for a "comprehensive } \\
\text { theory": tilting at } \\
\text { windmills? }\end{array}$ & $\begin{array}{l}\text { Entrepreneurship } \\
\text { Theory and } \\
\text { Practice, } 34(2) \text { : } \\
\text { p. } 249-260\end{array}$ & 2010 & 143 \\
\hline 21 & $\begin{array}{l}\text { Levie,J.D.; } \\
\text { Lichtenstein, } \\
\text { B.B. }\end{array}$ & $\begin{array}{c}\text { A terminal assessment of } \\
\text { stages theory: introducing a } \\
\text { dynamic states approach to } \\
\text { entrepreneurship }\end{array}$ & $\begin{array}{l}\text { Entrepreneurship: } \\
\text { Theory and } \\
\text { Practice, 34(2): } \\
\text { p.317-350 }\end{array}$ & 2010 & 224 \\
\hline 22 & $\begin{array}{l}\text { Masurel, E.; } \\
\text { Montfort, K.V. }\end{array}$ & $\begin{array}{l}\text { Life cycle characteristics of } \\
\text { small professional service } \\
\text { firms }\end{array}$ & $\begin{array}{l}\text { Journal of Small } \\
\text { Business } \\
\text { Management, } \\
\text { 44(3): p.461-473 }\end{array}$ & 2006 & 84 \\
\hline 23 & $\begin{array}{l}\text { Moores, K.; } \\
\text { Yuen, S. }\end{array}$ & $\begin{array}{c}\text { Management accounting } \\
\text { systems and organizational } \\
\text { configuration: a life-cycle } \\
\text { perspective } \\
\end{array}$ & $\begin{array}{c}\text { Accounting, } \\
\text { Organizations and } \\
\text { Society, 26(4-5): } \\
\text { p.351-389 } \\
\end{array}$ & 2001 & 414 \\
\hline 24 & $\begin{array}{l}\text { Phelps, R.; } \\
\text { Adams, R.; } \\
\text { Bessant, J. }\end{array}$ & $\begin{array}{l}\text { Life cycles of growing } \\
\text { organizations: a review with } \\
\text { implications for knowledge } \\
\text { and learning }\end{array}$ & $\begin{array}{l}\text { International } \\
\text { Journal of } \\
\text { Management } \\
\text { Reviews, 9(1): p1- } \\
30\end{array}$ & 2007 & 316 \\
\hline 25 & $\begin{array}{l}\text { Rutherford, } \\
\text { M.W.; Buller, } \\
\text { P.F.; McMullen, } \\
\text { P.R. }\end{array}$ & $\begin{array}{c}\text { Human resource } \\
\text { management problems } \\
\text { over the life cycle of small } \\
\text { to medium-sized firms } \\
\end{array}$ & $\begin{array}{l}\text { Human Resource } \\
\text { Management, } \\
\text { 42(4), p.321-335 }\end{array}$ & 2003 & 214 \\
\hline 26 & $\begin{array}{c}\text { Scott, M.; Bruce, } \\
\text { R. }\end{array}$ & $\begin{array}{c}\text { Five stages of growth in } \\
\text { small business }\end{array}$ & $\begin{array}{c}\text { Long Range } \\
\text { Planning, 20(3): } \\
\text { p.45-52 }\end{array}$ & 1987 & 947 \\
\hline 27 & $\begin{array}{c}\text { Simon, A.; } \\
\text { Bernardo, M.; } \\
\text { Karapetrovic, S.; } \\
\text { Casadesús, M. }\end{array}$ & $\begin{array}{c}\text { Integration of standardized } \\
\text { environmental and quality } \\
\text { management systems } \\
\text { audits } \\
\end{array}$ & $\begin{array}{l}\text { Journal of Cleaner } \\
\text { Production, 19(17- } \\
\text { 18): p.2057-2065 }\end{array}$ & 2011 & 79 \\
\hline 28 & $\begin{array}{l}\text { Skivington, J.E.; } \\
\text { Daft, R.L. }\end{array}$ & $\begin{array}{l}\text { A study of organizational } \\
\text { framework and process } \\
\text { modalities for the } \\
\text { implementation of business } \\
\text { level strategic decisions }\end{array}$ & $\begin{array}{c}\text { Journal of } \\
\text { Management } \\
\text { Studies, 28(1): } \\
\text { p.45-68 }\end{array}$ & 1991 & 157 \\
\hline 29 & Wendler, R. & $\begin{array}{l}\text { The maturity of maturity } \\
\text { model research: a } \\
\text { systematic mapping study }\end{array}$ & $\begin{array}{l}\text { Information and } \\
\text { Software } \\
\text { Technology, } \\
\text { 54(12): p.1317- } \\
\text { 1339 }\end{array}$ & 2012 & 125 \\
\hline 30 & Whetten, D.A. & $\begin{array}{l}\text { Organizational growth and } \\
\text { decline processes }\end{array}$ & $\begin{array}{c}\text { Annual Review of } \\
\text { Sociology, 13: } \\
\text { p.335-358 }\end{array}$ & 1987 & 450 \\
\hline
\end{tabular}

Fonte: Autoria própria (2018) 


\section{Estimar o Grau de Relevância dos Periódicos}

Para se determinar a relevância dos periódicos do portfólio bibliográfico analisou-se a quantidade de artigos que foram publicados por periódico dentro do portfólio bibliográfico como apresentado pelo Gráfico 1.

Gráfico 1 - Relevância dos Periódicos no Portfólio Bibliográfico

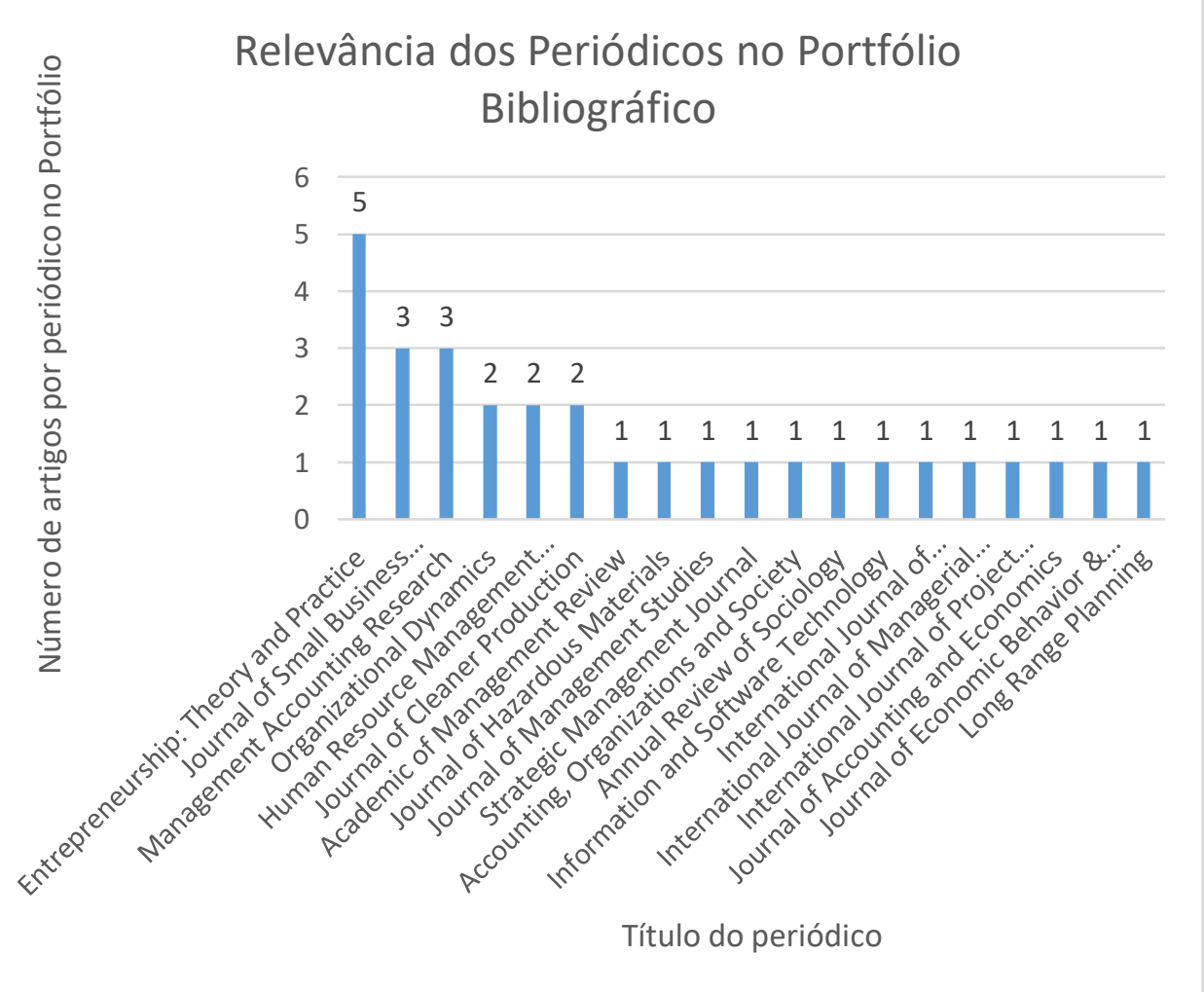

Fonte: Autoria própria (2018)

Os periódicos que apresentaram o maior número de artigos foram:

- Entrepreneurship: Theory and Practice - com 5 artigos;

- Journal of Small Business Management - com 3 artigos;

- Management Accouting Research - com 3 artigos.

A etapas de análise dos periódicos presentes nas referências dos artigos do portfólio bibliográfico foram suprimidas devido o foco adotado pela pesquisa ser analisar as publicações mais relevantes sobre o assunto.

\section{Relevância dos periódicos presentes nos artigos do portfólio bibliográfico.}

Nesta etapa da pesquisa, nos artigos do portfólio bibliográfico extrai-se os periódicos que os publicaram e busca-se analisar a relevância desses periódicos 
de acordo com a classificação Qualis CAPES (anos 2013 a 2016). O Gráfico 2, apresenta os periódicos por classificação Qualis-CAPES (2013 a 2016).

Foram identificados 19 periódicos onde estão publicados os artigos do portfólio bibliográfico. Verificou-se que do total 79\% (15 periódicos do total de 19) foram classificados como A1 e A2.

Gráfico 2 - Relevância dos Periódicos de acordo com a classificação QUALIS CAPES

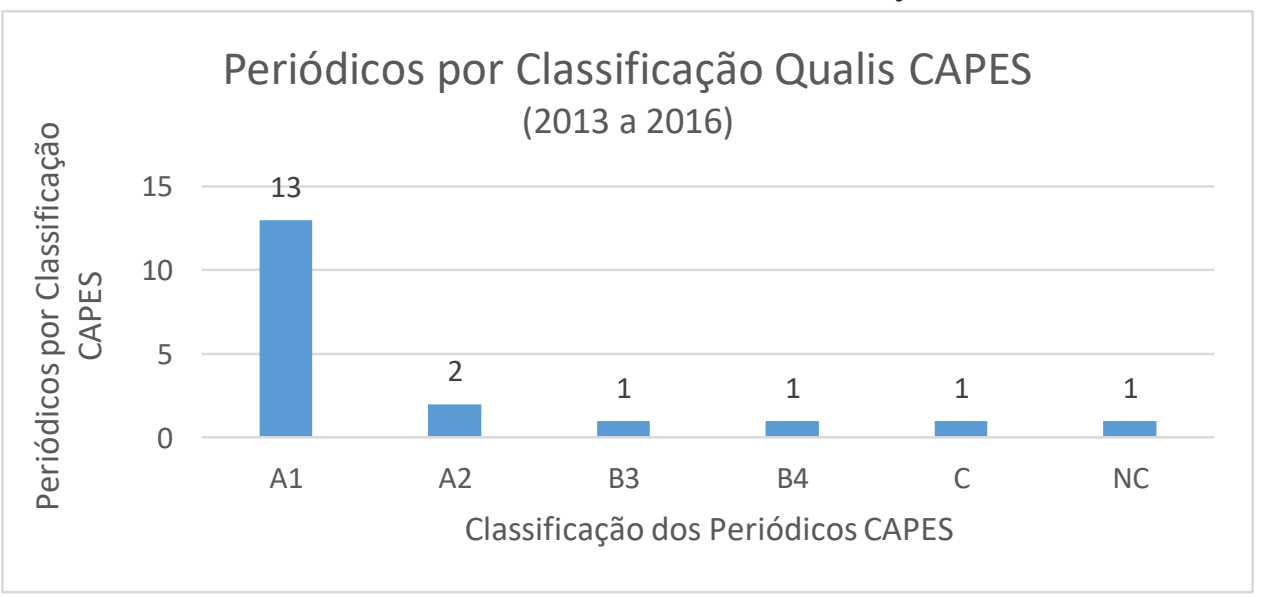

Fonte: Autoria própria (2018)

O outro passo desta etapa foi analisar a quantidade de publicações por periódico acordo com a classificação Qualis CAPES. Nesta identificou-se que $80 \%$ (24 artigos do total de 30) estão em periódicos classificados como A1 e A2. O Gráfico 3, apresenta as publicações por classificação Qualis-CAPES (anos 2013 a 2016).

Gráfico 3 - Número de Publicações de acordo com a classificação Qualis CAPES

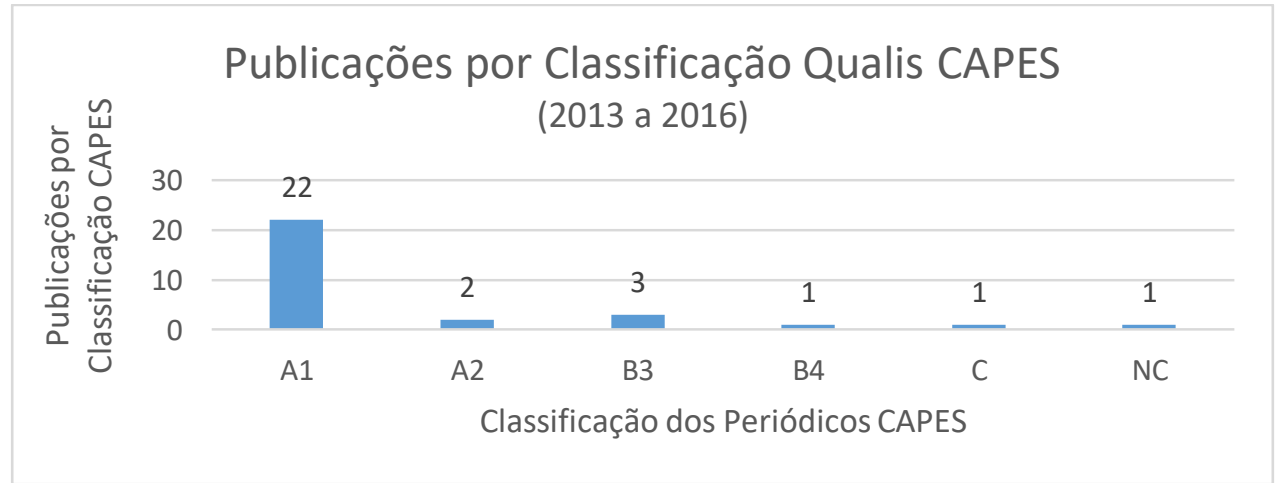

Fonte: Autoria própria (2018)

\section{Estimar o Reconhecimento Científico dos Artigos}

Para se determinar o reconhecimento científico utilizou-se como critério o número de citações que o artigo possui de acordo com as informações fornecidas pela ferramenta de pesquisa acadêmica Google Acadêmico.

Os dez artigos com maior número de citações e respectivamente seus números são apresentados no Gráfico 4 apresentado a seguir. 
No Gráfico 4, pode-se verificar o destaque especial que ocorre em relação aos três artigos com maior número de citações: "The evolution of firm networks: from emergence to early growth of the firm" (número 14 no Quadro 5), "Toward a descriptive stakeholder theory: An organizational life cycle approach" (número 17), "Five stages of growth in small business" (número 26), que representam juntos cerca de $50 \%$ das citações do grupo.

Gráfico 4 - Número de citações dos artigos do Portfólio Bibliográfico

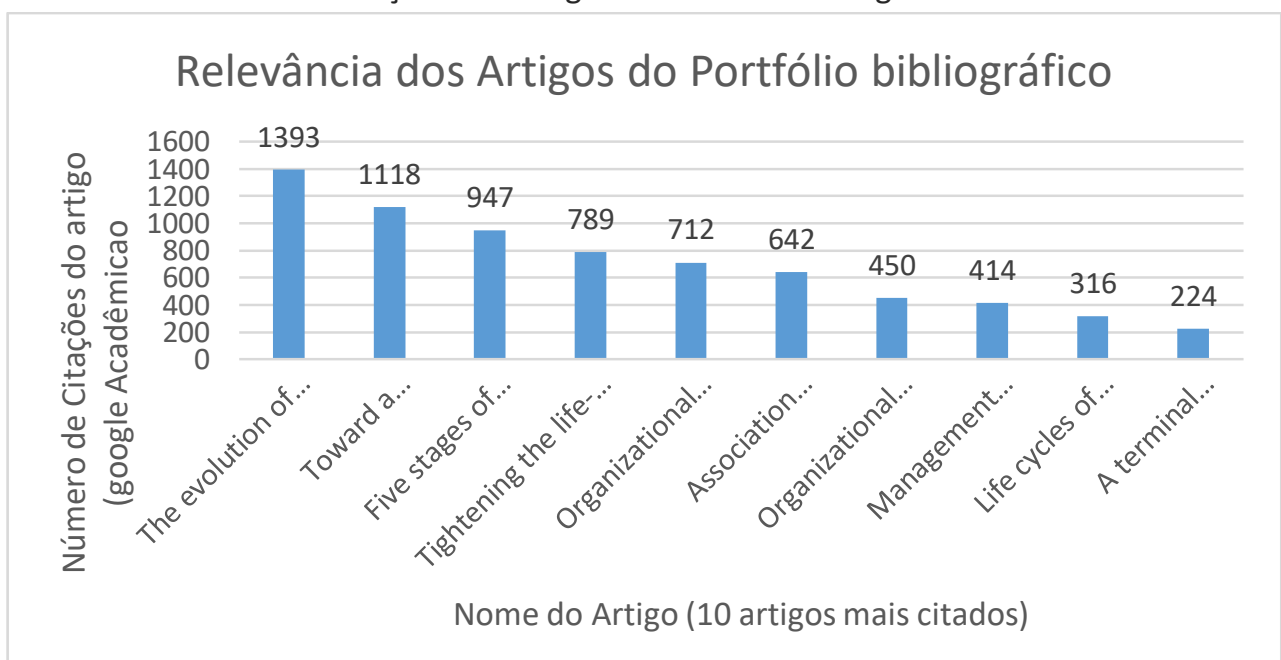

Fonte: Autoria própria (2018)

Outra análise realizada foi a verificação do número de citações dos artigos que compõe o portfólio bibliográfico, nos outros artigos presentes no portfólio, conforme recomendado pelo método Proknow-C (Ensslin et al., 2010) e apresentado no Gráfico 5.

Gráfico 5 - Número de citações das publicações dentro do Portfólio Bibliográfico

\section{Relevância de artigo nas referências do Portfólio Bibliográfico}

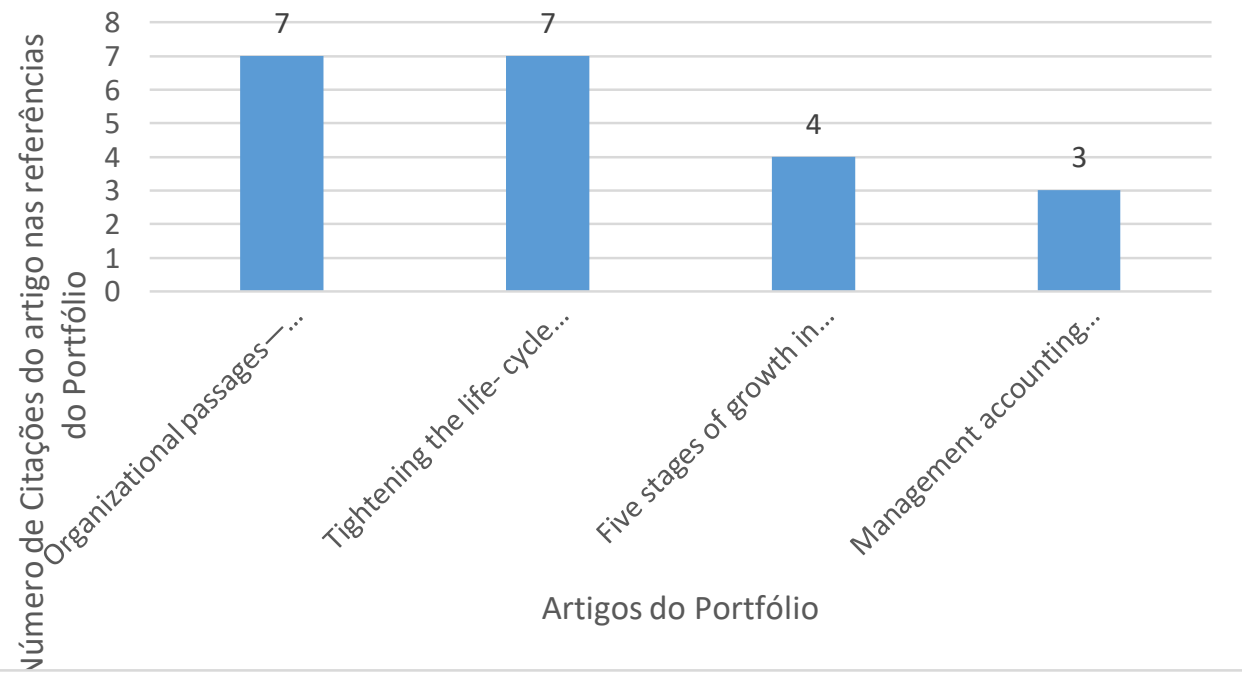

Fonte: Autoria própria (2018) 
Nessa análise identificaram-se dois artigos, que se destacaram pelo seguinte motivo: ambos tiveram sete citações dentro do portfólio bibliográfico, são eles: "Organizational passages - Diagnosing and treating lifecycle problems of organizations", o de número 2 e "Tightening the life-cycle construct: a taxonomic study of growth stage configurations in high-technology organizations" o de número 13 no Quadro 5.

\section{Estimar o Grau de Relevância dos Autores}

Para a avaliação do grau de relevância dos autores avalia-se os seguintes aspectos, conforme recomendado pelo método Proknow-C (Ensslin et al., 2010):

- Autores com maior número de publicações no portfólio bibliográfico;

- Autores com maior número de publicações nas referências do portfólio bibliográfico;

- Autores com maior destaque no portfólio bibliográfico.

Essas avaliações são descritas nos próximos subitens.

\section{Autores com maior participação no portfólio bibliográfico}

Somente dois autores apareceram em mais de uma publicação (duas publicações) sendo eles: Karapetrovic, S. e Casadesús, M., ambos da Universidade Alberta no Canadá, com as publicações sobre implementação de sistemas de gestão integrados.

\section{Autores com maior participação nas referências do portfólio bibliográfico.}

Analisou-se nesta etapa o número de vezes que um autor foi citado nas outras publicações dentro do portfólio bibliográfico, não necessariamente com a publicação constante no portfólio, mas considerou-se também outras possíveis publicações do autor.

Gráfico 6 - Número de citações das publicações dentro do Portfólio Bibliográfico

\section{Autores com maior número de citações nas} referências do portfólio bibliográfico

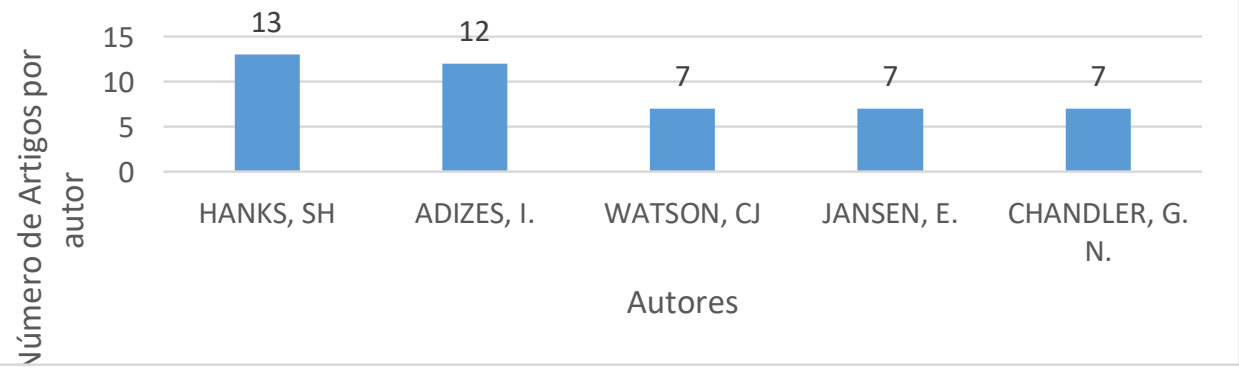

Fonte: Autoria própria (2018) 
Identificou-se em destaque os autores Hanks, S. H. com treze citações e o autor Adizes, I. com doze citações.

\section{Autores com maior destaque no portfólio bibliográfico}

Nessa etapa faz-se um cruzamento dos autores que mais possuem publicações que compõe o portfólio bibliográfico com os autores mais citados nas referências do portfólio bibliográfico.

Nesta etapa nenhum dos autores teve destaque no portfólio bibliográfico e nas referências dos artigos do portfólio.

\section{Determinar as Palavras-Chave mais usadas}

A determinação das palavras-chave mais usadas se dá pelo somatório das incidências dessas palavras nos artigos que compõe o portfólio bibliográfico. Os artigos mais antigos não apresentam as palavras-chave. Desta forma, foi realizada uma consulta ao Portal de Periódicos Capes, onde a ferramenta de busca apresenta na aba "detalhes" as palavras chave de todas a publicações de suas bases. 0 Gráfico 7 apresenta as palavras chave mais utilizadas.

Gráfico 7 - Palavras-chave com maior incidência no Portfólio Bibliográfico

\section{Palavras Chave com maior Incidência}

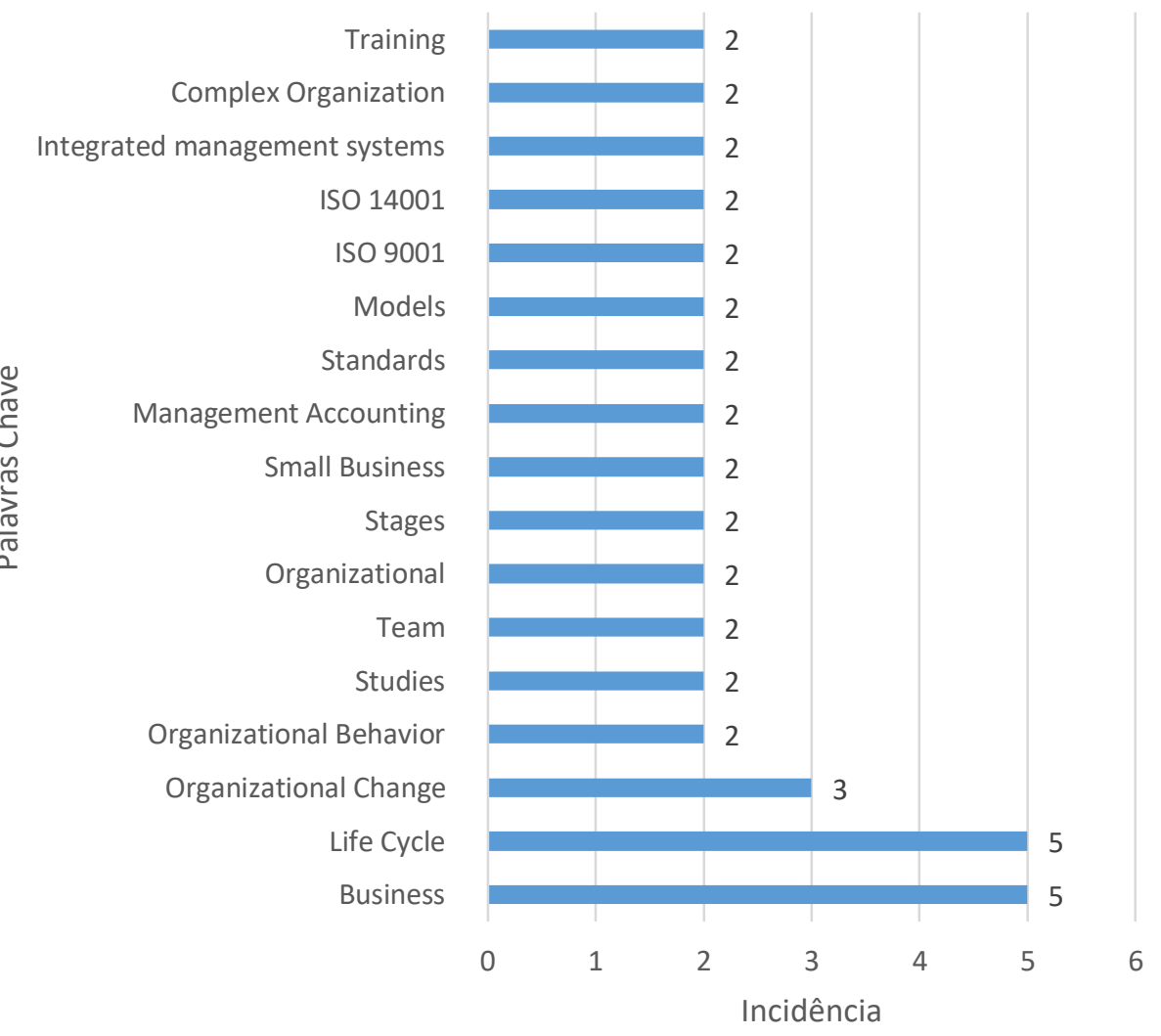

Fonte: Autoria própria (2018) 
Nessa etapa encerra-se a etapa de definição da formação do Portfólio Bibliográfico e respectivamente sua análise.

Nos próximos itens as publicações foram analisadas de acordo com o objetivo que os pesquisadores definiram, a fim de identificar possíveis lacunas de conhecimento sobre o assunto do trabalho.

\section{ANÁLISE SISTÊMICA DOS ARTIGOS DO PORTFÓLIO}

\section{Identificação dos critérios de análise}

Nesta etapa da pesquisa, analisou-se o portfólio bibliográfico a fim de extrair conceitos ou lacunas do conhecimento a serem exploradas por outras pesquisas, sendo esta análise do conteúdo dos artigos, segundo os critérios de análise definidos pelos pesquisadores (ENSSLIN et al., 2010).

Os critérios de análise são apresentados no Quadro 6. A partir dos critérios explicitados, os artigos foram analisados conforme apresentado no item posterior.

Quadro 6 - Identificação dos critérios de análise

\begin{tabular}{|c|c|c|}
\hline $\mathbf{N}^{\circ}$ & Critério & O que busca \\
\hline 1 & $\begin{array}{c}\text { Ciclo de Vida } \\
\text { Organizacional }\end{array}$ & $\begin{array}{c}\text { Qual o conceito de Ciclo de Vida Organizacional } \\
\text { tratado na publicação? }\end{array}$ \\
\hline 2 & $\begin{array}{c}\text { Modelo de Ciclo de Vida } \\
\text { Organizacional }\end{array}$ & $\begin{array}{c}\text { Qual a abordagem teórica tratado nas } \\
\text { publicações? }\end{array}$ \\
\hline 3 & $\begin{array}{c}\text { Fases do Ciclo de Vida } \\
\text { Organizacional }\end{array}$ & $\begin{array}{c}\text { Qual o número de fases, características e atributos } \\
\text { presentes nos modelos de ciclo de vida } \\
\text { organizacional apresentados nos artigos do } \\
\text { portfólio? }\end{array}$ \\
\hline 4 & $\begin{array}{c}\text { Estágio atual, avanço ou } \\
\text { retrocesso nas fases do } \\
\text { Ciclo de Vida } \\
\text { Organizacional }\end{array}$ & $\begin{array}{c}\text { Como se dá a definição do estágio atual, o avanço } \\
\text { ou retrocesso nas fases do Ciclo de Vida } \\
\text { Organizacional? }\end{array}$ \\
\hline 6 & $\begin{array}{c}\text { Implantação de Sistemas de } \\
\text { Gestão }\end{array}$ & $\begin{array}{c}\text { As publicações abordam o processo de } \\
\text { implantação de Sistemas de Gestão? }\end{array}$ \\
\hline 7 & Mensuração & $\begin{array}{c}\text { As publicações apresentam propostas de } \\
\text { mensuração do desempenho da empresa após } \\
\text { implantação de Sistemas de Gestão? }\end{array}$ \\
\hline 8 & Antegração & $\begin{array}{c}\text { Os modelos propostos nas publicações relacionam } \\
\text { Ciclo de Vida Organizacional e Implantação de } \\
\text { Sistemas de Gestão }\end{array}$ \\
\hline
\end{tabular}

Fonte: Autoria própria (2018)

\section{Análise dos artigos segundo os eixos de avaliação}

De acordo com os critérios definidos, foi realizada a análise dos conteúdos dos artigos do portfólio bibliográfico e as informações foram extraídas e 
compiladas no quadro de análise apresentado no Quadro 7 que apresenta as oportunidades de pesquisa conforme os critérios de análise.

Quadro 7 - Oportunidade de Pesquisa de acordo com os critérios de análise

\begin{tabular}{|c|c|c|}
\hline $\mathbf{N}^{\circ}$ & Critério & Oportunidade de Pesquisa \\
\hline 1 & $\begin{array}{l}\text { Ciclo de Vida } \\
\text { Organizacional }\end{array}$ & $\begin{array}{l}\text { Analisar se os conceitos de Desenvolvimento, } \\
\text { Crescimento e Declínio das empresas podem ser } \\
\text { analisados pela Teoria do Ciclo de Vida Organizacional }\end{array}$ \\
\hline 2 & $\begin{array}{l}\text { Modelo de Ciclo de } \\
\text { Vida Organizacional }\end{array}$ & $\begin{array}{c}\text { Como desenvolver um modelo de desenvolvimento } \\
\text { organizacional que comtemple a empresa e o mercado } \\
\text { em que ela atue? }\end{array}$ \\
\hline 3 & $\begin{array}{l}\text { Fases do Ciclo de Vida } \\
\text { Organizacional }\end{array}$ & $\begin{array}{c}\text { É possível predefinir as fases do ciclo de vida } \\
\text { organizacional de uma empresa? }\end{array}$ \\
\hline 4 & $\begin{array}{l}\text { Estágio atual, Avanço } \\
\text { ou retrocesso nas fases } \\
\text { do Ciclo de Vida } \\
\text { Organizacional }\end{array}$ & $\begin{array}{c}\text { A partir da relação empresa e mercado em que esta } \\
\text { empresa atua é possível entender os motivos dos } \\
\text { avanços ou retrocessos do desenvolvimento } \\
\text { organizacional? }\end{array}$ \\
\hline 5 & $\begin{array}{l}\text { Implantação de } \\
\text { Sistemas de Gestão }\end{array}$ & $\begin{array}{c}\text { Como implantar os elementos de gestão necessários para } \\
\text { uma organização de acordo com sua fase atual de } \\
\text { desenvolvimento? }\end{array}$ \\
\hline 6 & Mensuração & $\begin{array}{c}\text { Como criar um sistema de mensuração do desempenho } \\
\text { organizacional para auxiliar a gestão antecipar suas } \\
\text { decisões? }\end{array}$ \\
\hline 7 & Integração & $\begin{array}{c}\text { Como criar um modelo que integre as diversas fases da } \\
\text { empresa com o tipo de empresa, perfil gerencial e o } \\
\text { mercado em que atue? }\end{array}$ \\
\hline 8 & Aperfeiçoamento & $\begin{array}{l}\text { Como gerar um modelo que apresenta a situação atual da } \\
\text { empresa e proposta para a melhoria do desempenho e a } \\
\text { sistematização das antecipações gerenciais? }\end{array}$ \\
\hline
\end{tabular}

Fonte: Autoria própria (2018)

\section{CONCLUSÃO}

Este trabalho contribui para apresentar algumas lacunas ainda a serem analisadas em relação à temática desenvolvimento organizacional. Como apresentado, ainda não houve constatações empíricas das várias propostas de desenvolvimento organizacional apresentado pela corrente de pesquisa de ciclo de vida organizacional.

Concordando com Whetten (1987), verificou-se que as pesquisas com filiação teórica no ciclo de vida organizacional continuam focando, grande parte, nas fases iniciais do desenvolvimento organizacional, porém ainda existem lacunas sobre como gerenciar o crescimento organizacional e as fases de declínio organizacional, uma vez que, esse conhecimento pode vir auxiliar gestores nesses momentos mencionados.

Outro ponto a avançar é a aproximação dos conceitos e variáveis de análise entre pesquisadores e gestores, as publicações evidenciaram que o enquadramento teórico nem sempre consegue expressar o que os gestores das organizações entendem como desenvolvimento organizacional. 
Algumas pesquisas empíricas identificaram que para o estudo do desenvolvimento organizacional pelo menos três variáveis devem ser analisadas: a empresa, o mercado em que ela atua e a equipe gerencial ou o empreendedor.

Por fim, percebeu-se que ainda há uma lacuna de conhecimento sobre quais os elementos específicos necessários para um eficiente gerenciamento organizacional que antecipe crises organizacionais e como implementá-los nas empresas que ainda não os possuem. Existem vários nichos de pesquisa cientifica que podem ser exploradas pelos cientistas das áreas de gestão, engenharias e correlatas. 


\title{
Implementation of management systems in accordance with the organizational life cycle: a systematic review of literature
}

\begin{abstract}
One way of analysing a given subject in academia is through a Systematic Review of Literature. There are several methods that consists of a structured process that allows the identification of knowledge gaps according to the definitions that have been made for this type of research on the subject in question. The objective of this paper is to analyse scientific references about the implementation of business management systems according to the organizational life cycle. For this, he used the ProKnow-C method to quantitatively search and analyse articles relevant for this topic. The definitions of the databases were made in December 2016. A total of 6,311 articles were identified, of which 30 most relevant articles were selected, published between the years of 1979 and 2012 . The analysis of the publications showed that there is potential for continuity of research in subjects discussed for years, such as Organizational Life Cycle Theory, and new correlated themes, such as the Organizational Performance Measurement and the Systematization of Managerial Advances.
\end{abstract}

Implementation of Management Systems. Organizational Life Cycle. Systematic Review of Literature. ProKnow-C. 
ACHTENHAGEN, L.; NALDI, L.; MELIN, L. "Business growth": do practitioners and scholar really talk about the same thing? Entrepreneurship Theory and Practice, v.34, i.3, p.289-316, 2010. http://dx.doi. org/10.1111/j.1540-6520.2010.00376.x crossref

ADIZES, I. Organizational passages: diagnosing and treating lifecycle problems of organizations. Organizational Dynamics, v.8 , i.1, p.3-25, 1979. crossref

CANTNER, U.; WOLF, T. The selective nature of innovator networks: from the nascent to the early growth phase of the organizational life cycle. p.175-204. 2018. In: CUBICO, S.; FAVRETTO, G.; LEITÃO, J.; CANTNER, U. (eds) Entrepreneurship and the industry life cycle. Studies on Entrepreneurship, Structural Change and Industrial Dynamics. Springer, Cham. https://doi.org/10.1007/978-3-319-89336-5_8. crossref

ENSSLIN, L.; LACERDA, R.T.O.; TASCA, J.E. ProKnow-C: Knowledge Development Process-Constructivist: processo técnico com patente de registro pendente junto ao INPI. Brasil: [sn], 2010.

GREENHALGH, T. Papers that summarize other papers (systematic review and meta-analyses). British Medical Journal, London, v. 315, i.7109, p. 672-675, 1997. crossref

LEVIE, J.; LICHTENSTEIN, B.B. A terminal assessment of stages theory: introducing a dynamic states approach to entrepreneurship. Entrepreneurship Theory and Practice, v.34, i.2, p.317-350, 2010. crossref

LI, Y.; ZHANG, X. How does firm life cycle affect board structure? Evidence from China's listed privately owned enterprises. Management and Organization Review, v.14, i.2, p.305-341, 2018. https://doi.org/10.1017/mor.2017.55. crossref LIPPITT, G.L.; SCHMIDT, W.H. Crises in a developing organization. Harvard Business, v.45, i.6, p.102-112, 1967.

MORIOKA, S. N.; IRITANI, D. R.; OMETTO, A. R.; CARVALHO, M. M. Revisão sistemática da literatura sobre medição de desempenho de sustentabilidade corporativa: uma discussão sobre contribuições e lacunas. Gestão \& Produção, v.25, n.2, p.284-303. 2018. https://dx.doi.org/10.1590/0104-530x2720-18. crossref 
PHELPS, R.; ADAMS, R.; BESSANT, J. Life cycles of growing organizations: A review with implications for knowledge and learning. International Journal of Management Reviews, v.9, i.1, p.1-30, 2007. https://doi.org/10.1111/j.14682370.2007.00200.x. crossref

ROCCO, P.; MANNA, R. The need for requisite variety to support growth: an organizational life cycle perspective. Journal of Strategy and Management, v.11, i.2, p.241-256, 2018. https://doi.org/10.1108/JSMA-10-2016-0072 . crossref

SILVEIRA, L. M.; PETRINI, M. Desenvolvimento Sustentável e Responsabilidade Social Corporativa: uma análise bibliométrica da produção científica internacional. Gestão \& Produção, v.25, i.1, p.56-67, 2018. https://dx.doi.org/10.1590/0104-530x3173-16. crossref

TOMOMITSU, H. T. A.; CARVALHO, M. M.; MORAES, R. O. A evolução da relação entre a gestão de projetos e a gestão do conhecimento: um estudo bibliométrico. Gestão \& Produção, v.25, i.2, p.354-369, 2018. https://dx.doi.org/10.1590/0104$530 \times 3150-16$. crossref

VILELA, L.O. Aplicação do Proknow-C para seleção de um portifólio bibliográfico e análise bibliométrica sobre avaliação de desempenho da gestão do conhecimento. Gestão Industrial, v.8, n.1, p.76-92, 2012. https://doi.org/10.3895/S1808-04482012000100005. crossref

WHETTEN, D.A. Organizational Growth and decline process. Annual Review of Sociology, v.13, p.335-358, 1987.

https://doi.org/10.1146/annurev.so.13.080187.002003. crossref

Como citar:

SÁTIRO, R.S.; SERRA, S.M.B. Implementação de sistemas de gestão de acordo com o ciclo de vida organizacional: uma revisão sistemática da literatura. R. Gest. Industr., Ponta Grossa, v. 15, n. 4, p. 200 220, Out./Dez. 2019. Disponível em: http://periodicos.utfpr.edu.br/revistagi. Acesso em: 2019. Correspondência:

Rodrigo Salgado Sátiro e Sheyla Mara Baptista Serra

Programa de Pós-graduação em Engenharia Civil (PPGECiv), Universidade Federal de São Carlos (UFSCar), Rodovia Washington Luis Km 235, São Carlos, SP, Brasil.

Direito autoral: Este artigo está licenciado sob os termos da Licença Creative Commons-Atribuição 4.0 Internacional.

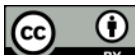

